\title{
A Comparison of the Changes in Gestational Weight, Body Mass Index, and Serum Vitamin D Level in Gestational Diabetes Mellitus Patients Complemented with Vitamin D in Contrast to Those Who Did Not Receive the Supplement: A Protocol for Systematic Review and Meta-Analysis of Randomised Controlled Trials
}

\author{
Sumanta Saha ${ }^{a}$ Sujata Saha ${ }^{b}$ \\ ${ }^{a}$ National Institute for Research in Tuberculosis, Chennai, India; ${ }^{b}$ Mankar College, Mankar, India
}

\section{Keywords}

Body mass index · Gestational diabetes · Pregnancy weight gain · Vitamin D · Dietary supplement

\begin{abstract}
Aims: To date, it is largely unknown how body mass index (BMI), gestational weight (GW), and stored vitamin $\mathrm{D}(25(\mathrm{OH})$ D) levels change in the vitamin D-supplemented gestational diabetes mellitus (GDM) patients, irrespective of their prepregnancy BMI. Therefore, to study this association, a prospective systematic review and meta-analysis protocol is proposed here. Methods: Primarily, different databases (PubMed, Embase and Scopus) will be searched (without any limitation to date or language) for randomised parallel-arm trials comparing GW, BMI and stored vitamin D level in the body among GDM patients who were supplemented and not supplemented with vitamin $D$, besides their conventional GDM care. The study selection process and the consecutive risk of bias assessment will adhere to the PRISMA 2009
\end{abstract}

flow chart and the Cochrane collaboration's guideline, respectively. These interventions' effect on the respective outcomes will be compared by meta-analysis (along with an assessment of the statistical heterogeneity) when comparable data will be available from at least 4 studies. Subgroup analysis and Egger's meta-regression will follow if an adequate number of trials are available. Narrative reporting will be considered when a quantitative comparison is not feasible for any of the outcomes. Conclusion: The proposed review aims to compare the BMI, GW, and 25(OH)D levels in the blood between the vitamin $D$ supplemented and not supplemented GDM patients.

(C) 2019 The Author(s)

Published by S. Karger AG, Basel

Registration: We have submitted this protocol to the PROSPERO (https://www.crd.york.ac.uk/PROSPERO/) for registration (presently under assessment).

\begin{tabular}{|c|c|c|}
\hline KARGER & $\begin{array}{l}\text { (c) } 2019 \text { The Author(s) } \\
\text { Published by S. Karger AG, Basel }\end{array}$ & $\begin{array}{l}\text { Karger } \\
\text { Open access }\end{array}$ \\
\hline $\begin{array}{l}\text { E-Mail karger@karger.com } \\
\text { www.karger.com/ijd }\end{array}$ & $\begin{array}{l}\text { This article is licensed under the Creative } \\
\text { NonCommercial-NoDerivatives } 4.0 \text { Intern } \\
\text { NC-ND) (http://www.karger.com/Servic } \\
\text { Usage and distribution for commercial pur } \\
\text { tribution of modified material requires writ }\end{array}$ & $\begin{array}{l}\text { Commons Attribution- } \\
\text { ational License (CC BY- } \\
\text { es/OpenAccessLicense). } \\
\text { poses as well as any dis- } \\
\text { ten permission. }\end{array}$ \\
\hline
\end{tabular}

Dr. Sumanta Saha

A- $10 / 323$

Kalyani, West Bengal 741235 (India)

E-Mail sumanta.saha@uq.net.au 


\section{Introduction}

Glucose intolerance (to any degree), when started or recognised for the first time during pregnancy, is referred to as gestational diabetes mellitus (GDM) [1]. GDM diagnosis (between 24 and 28 weeks of pregnancy) comprises of a screening test with a $50-\mathrm{g}, 1-\mathrm{h}$ oral glucose challenge test, and a successive $100-\mathrm{g}, 3$-h oral glucose tolerance test (when the former test's result is abnormal) [1]. Two or more abnormal test results confirm the diagnosis [1]. Currently, the number of GDM cases is rising parallel to the escalating trend of obesity and a sedentary lifestyle [2]. In 2017, globally, GDM alone caused almost $86 \%$ of hyperglycaemic conditions during pregnancy and affected more than 18 million live births in some form [3]. To achieve glycaemic control, the traditional management of GDM begins with lifestyle interventions like dietary modifications, exercise, and blood glucose monitoring $[1,4]$. Insulin treatment is started when these approaches fail to attain optimal glycaemic control $[1,4]$.

Despite these treatments, complications in GDM pregnancies are not uncommon. The vast array of complications that can affect both the GDM mother (e.g., pre-eclampsia, polyhydramnios, type 2 diabetes risk) [5] and her newborn (e.g., macrosomia, metabolic syndrome risk in the infant) [5] have instigated research on the possible role of vitamin D supplementation in decreasing such unwanted events. Such research is also inspired by the novel evidence that suggests GDM's association with vitamin D insufficiency [6-10] and a decline in the GDM prevalence on prenatal vitamin D supplementation [11, 12].

Vitamin D (calciferol) is a fat-soluble vitamin. It is available from diet and supplements in two inactive forms - vitamin D2 (ergocalciferol) and D3 (cholecalciferol) $[13,14]$. Cholecalciferol is also synthesised in the skin (by sun exposure) [13]. After hydroxylation in the liver and consecutively in the kidney, calcitriol $(1,25(\mathrm{OH}) 2$ D), the active form of vitamin D, is formed [13]. Calcitriol, consequent to binding to the vitamin D receptor (VDR) (a hormonal receptor in the cell nucleus), stimulates intestinal absorption of calcium and phosphorus [14]. It also plays a role in calcium reabsorption via the kidney [14]. Besides the role in bone metabolism, vitamin $\mathrm{D}$ (via its active calcitriol form) plays a physiologic role in pregnancy through the VDR located in the uteroplacental tissue [15]. In GDM, excessive production and expression of a VDR-modulating enzyme gene CYP24A1 (that has a negative correlation with the serum vitamin $\mathrm{D}$ levels) in

Vitamin D Supplementation in GDM Patients the placental tissue lowers the vitamin D level $[15,16]$. At a molecular level, plausibly due to the diminished vitamin $D$ levels in the GDM mothers, VDRs are up-regulated in the extra-villous trophoblasts and placental endothelium [17].

Contemporarily, several clinical trials have studied the effects (e.g., biomarkers of inflammation, oxidative stress, cardiometabolic risk, and pregnancy outcomes) of vitamin D supplementation in GDM patients [18-21]. Although the role of vitamin D complementation in GDM patients has been tested widely, little is known about how it affects their body mass index (BMI) and gestational weight (GW).

In this regard, the existing research has primarily investigated the association between GDM and its adverse outcomes (maternal and neonatal) in relation to prepregnancy BMI. Pre-pregnancy BMI is a risk factor of GDM, particularly among overweight (BMI $25 \mathrm{~kg} / \mathrm{m}^{2}$ or more) or obese (BMI $30 \mathrm{~kg} / \mathrm{m}^{2}$ or more) women [22]. High BMI values can induce chronic low-grade inflammation, which subsequently raises the susceptibility to GDM development [1]. Besides the pre-pregnancy BMI, the gestational weight gain (GWG), especially in early pregnancy, is another predictor of GDM risk [23]. Disproportionate GWG in GDM patients can cause adverse maternal outcomes (caesarean section) and neonatal outcomes (poor APGAR score, large for gestational age and macrosomia) $[24,25]$. Recent evidence suggests that preconceptional BMI and excessive GWG are independent risk factors in GDM patients [24]. However, it is not clear how BMI and GW changes in vitamin D-supplemented and not supplemented GDM patients irrespective of their pre-pregnancy BMI. To this end, the updated GWG guidelines (2009) by the Institute of Medicine offer little help, as they are chiefly based on pre-pregnancy BMI categories, and not based on the GDM subpopulation [25, 26].

Additionally, for optimal functioning of the vitamin D supplements in GDM patients, it is vital to ensure that vitamin $\mathrm{D}$ attains a proper blood level. Therefore, it is important to research if such supplementation favours a rise in the blood 25(OH)D levels (an ideal marker for measuring the stored levels of ingested vitamin D) [27]. While $25-80 \mathrm{ng} / \mathrm{mL}$ of $25(\mathrm{OH}) \mathrm{D}$ is the optimal level, levels of $<30 \mathrm{ng} / \mathrm{mL}$ and $<20 \mathrm{ng} / \mathrm{mL}$ are considered as vitamin $\mathrm{D}$ insufficiency and vitamin D deficiency, respectively [27].

Based on this background, to study these unexplored effects of vitamin D supplementation in GDM patients, this systematic review protocol is proposed. The chief aim of this prospective review is to compare the GW, BMI, 
and blood 25(OH)D levels between GDM patients supplemented and not supplemented with vitamin D antenatally.

\section{Methods}

This protocol is built on the guidelines recommended in the PRISMA-P checklist [28]. The inclusion criteria of the proposed systematic review are as the following. Randomised parallel-arm (any number) clinical trials will be eligible for inclusion. Trial participants should be pregnant females of any age and any pre-pregnancy BMI who have been diagnosed with GDM during their current gestation. The mean BMI $\left(\mathrm{kg} / \mathrm{m}^{2}\right), \mathrm{GW}(\mathrm{kg})$ and blood $25(\mathrm{OH})$ D levels $(\mathrm{ng} / \mathrm{mL})$ of the participants should have been reported by the trialists both at the baseline and at the end of the trial (before childbirth) among the participants receiving and not receiving vitamin D supplementation, besides their regular GDM care. The diagnosis and treatment of GDM and the dosage, regimen and duration of the therapy with the vitamin $\mathrm{D}$ supplementation will be accepted as per the trialists. In the intervention group, vitamin D may be the sole supplement or used alongside other supplements, as described by the investigators, whereas the comparator group may receive no supplement or placebo or any other supplementation except vitamin D.

History of diabetes other than GDM (i.e., type 1 or type 2 diabetes), trials of any other design besides the above-described ones (e.g., cross-over trials, observational studies), and animal studies will constitute the exclusion criteria.

By searching the different electronic databases (PubMed, Embase and Scopus), we will attempt to identify the qualifying trials. These searches will not be restricted to any date or language. Additionally, the eligible trials will be searched in the bibliography of the papers included in the proposed review.

The following is the draft database search strategy to be used in the PubMed to search the titles and abstracts of eligible trials: "vitamin D" OR calciferol OR "vitamin D2" OR ergocalciferol OR "vitamin D3" OR cholecalciferol AND "gestational diabetes" OR GDM. The search will also incorporate the following MeSH terms: "vitamin d3," "vitamin d2," and "vitamin d." The filter "Clinical trial" will be applied to all searches. Similar search terms will be used for the other databases. If suitable filters for searching the randomised trials are unavailable in a database, the following search terms will also be used: "trial" OR "randomised" OR "randomized" OR "controlled." The database search will be performed by the first author.

Search results across the databases will be collated using a reference handling software and uploaded to Rayyan [29] systematic reviews software for eliminating duplicate papers and selecting papers requiring full-text review. Then, the review authors will independently skim through the titles and abstracts of the search results to find trials matching the afore-mentioned eligibility criteria. Subsequently, research papers will be selected for full-text independent reading by the review authors if they seem to match the proposed eligibility criteria or if making a definitive decision about inclusion or exclusion is difficult. A record of studies excluded from the review following the full-text reading will be maintained with an explanation for exclusion. Throughout the study selection process, any conflict in opinion between the review authors will be resolved by discussion. The entire study selection process will adhere to the PRISMA 2009 flow diagram [30].

Next, the first author will extract the data (relevant to study design, trial population, intervention received by the intervention group and comparator group, and the outcomes of interest) from the recruited trials, which will be then checked by the coauthor. Subsequent study design-related information will be retrieved: randomisation details, number of intervention arms, multi-centre or single-centre trial, trial duration, participant consent, funding, and trial identification number. The trial participants' data that will be collected are the following: diagnosis, number of participants receiving each of the interventions, mean age of the trial population, the frequency of participants for whom outcome data are not available, and the nation/s from where the trial participants originated. Regarding the interventions, their types, dosages, and regimens for the respective intervention groups will be extracted. Lastly, data relevant to the outcome both at baseline and at follow-up (post-intervention) will be collected.

Using the Cochrane Collaboration's tool, the outcome level risk of bias of the reviewed trials will be assessed for selection bias, performance bias, detection bias, attrition bias, reporting bias and other biases [31].

Each of these biases's risk will be categorised as low, high, or unclear (when it will not be possible to affirmatively decide if the risk of bias is low or high) based on the review authors' assessment. The authors will evaluate the risk of bias independently in a blinded manner and then compare their findings; all differences in opinion will be settled by discourse. Trials with a high risk of bias component will not be incorporated into the metaanalysis.

The intervention effects on individual outcomes will be juxtaposed using meta-analysis on an available case analysis basis. Because of the continuous nature of the outcome data, the summary effect will be determined in either the mean differences or standardised mean differences, depending on the measuring scale's similarity or dissimilarity, respectively. All of the metaanalyses (inverse variance method) will use the endpoint means and their standard deviations (SD). For more than two-arm trials, a combined mean and its SD will be calculated for the respective treatment arms. If the trials are clinically heterogeneous, the meta-analysis will use a random-effects model. Alternatively, a fixed-effects model shall be preferred. The clinical heterogeneity will be determined using the basic trial characteristics, such as the diverseness among the participants, investigators and study design. A random-effects model meta-analysis will be accompanied by predictive interval calculation to see how the respective outcomes will differ between the intervention groups in future trials. The summary estimates' statistical significance will be assessed at a $p$ value $<0.05$ at a $95 \%$ confidence interval (CI).

If the endpoint means' SD is unavailable, the consequent steps will be followed. First, the trialists will be contacted by the corresponding author by email for these SD values. On non-receipt of any reply to the email in the subsequent 7 days, the SD will be calculated algebraically from the standard errors (SE) or CIs of the follow-up means. On failure of this method, SE, CI, $t$ values or $p$ values of differences in the between-group means will be used to derive the $\mathrm{SD}$. If the above methods prove futile, the SD change 
for mean change from baseline will be determined by an imputation method. For an outcome, when the SD is imputed at a minimum for 1 trial, the meta-analysis for that outcome will be performed for mean changes from baseline. The imputation method will be substituting the baseline SD for the mean as the endpoint $\mathrm{SD}$ of the average and vice versa. Consequently, the mean change from baseline will be calculated using a correlation coefficient of 0.5 .

Using the $I^{2}$ statistics and the $p$ value of Cochrane's Q, the statistical inconsistency among the trials will be estimated. The heterogeneity will be categorised as low, moderate and high based on an approximate $I^{2}$ value of 25, 50, and 75\%, respectively [32]. The statistical significance of the $p$ value of Cochran's $Q$ will be determined at a $p$ value of $<0.1$ [31]. When a meta-analysis of at least 10 trials shows the statistical inconsistency of the summary estimate, a subgroup analysis will follow to compare the outcome between women older than 35 years (mean age) and less than 35 years (mean age). The rationale behind this analysis is the fact that GDM prevalence is often higher with advanced maternal age (i.e., >35 years of age) $[33,34]$. Funnel plot, in conjunction with the Egger's meta-regression test, will be used to examine the chances of publication bias when a meta-analysis is performed with at least 10 trials.

Finally, depending on the type of data that will be available from the reviewed trials, 4 types of sensitivity analysis are proposed here for each of the outcomes. The first type will repeat the meta-analysis for the respective outcomes using an alternative model (fixed-effects or random-effects) based on what was used in the preliminary meta-analysis. The second variant will repeat the meta-analysis for respective outcomes by excluding a trial each time. The third type will repeat the meta-analysis for the respective outcomes and will include trials with at least 1 high risk of bias component (if any). Finally, in the last type, if an SD imputation is required during the primary meta-analysis, it will be repeated with the correlation coefficient values of 0.3 and 0.8 .

Data will be prepared for meta-analysis in Microsoft's excel software, and all meta-analysis-related analyses will be conducted in Stata statistical software (StataCorp, College Station, TX, USA).

For any outcome, when fewer than 4 trials are available for meta-analysis, the findings will be reported narratively. Utilising the GRADE approach, the quality of evidence will be assessed for the outcomes that will be statistically significantly different between the contrasted interventions [35].

\section{Discussion}

The primary strength of the planned systematic review is its uniqueness in the context discussed above. Plausibly, this will be the first systematic review investigating how vitamin D supplementation can affect BMI, GW and serum 25(OH)D levels in GDM patients irrespective of their pre-pregnancy BMI. Furthermore, the inclusion of randomised controlled trials only (considered as the highest level of epidemiological evidence) will perhaps increase the rigour of the synthesised evidence. Besides, the intended database search for finding eligible trials is likely to be comprehensive as it will not be restricted to any date or language.

Despite these strengths, the intended evidence synthesis effort of the proposed systematic review is prone to certain limitations. The review will not be able to provide any BMI category-specific (e.g., for overweight or obese) evidence as it will recruit trial population irrespective of their pre-conceptional BMI. Additionally, the findings of the prospective review will be at risk of bias if the GDM diagnostic criteria used across the trials are quite different (since the diagnosis of GDM will be accepted as per the trialists). Likewise, it might not be possible to make a comparison of the outcomes (of interest) in the GDM patients based on specific dosage, regimen and duration of consumption of vitamin D supplementation if these components are remarkably dissimilar across the trials.

Finally, regarding the implication of the contemplated study, gynaecologists and future trialists may use it as a preliminary source of synthesised evidence in the context. Since BMI is an important determinant of GDM [22], knowledge regarding its changes after vitamin D supplementation might prove useful for obstetricians to anticipate the expected change in BMI while treating GDM patients to provide better obstetric care. Additionally, it will perhaps provide some insight into whether adequate vitamin D levels are attainable in the blood of GDM patients following vitamin D supplementation during pregnancy.

\section{Conclusion}

The prospective systematic review will compare the GDM patients' BMI, GW, and $25(\mathrm{OH}) \mathrm{D}$ level in the blood between the recipients and non-recipients of vitamin $\mathrm{D}$ antenatally.

\section{Acknowledgement}

This paper has been prepared independently by the authors and is not related to their affiliated institutions.

\section{Statement of Ethics}

As no human subjects will be involved in the proposed systematic review, an ethical approval will not be needed. 


\section{Disclosure Statement}

The authors have no conflicts of interest to declare.

\section{Author Contributions}

The conception, designing, and manuscript writing of this paper were primarily done by the first author. The coauthor rechecked the draft and also hard edited the manuscript.

\section{Funding Sources}

No funding was received for this work.

\section{References}

1 Quintanilla Rodriguez BS, Mahdy H. Gestational Diabetes. StatPearls [Internet]. Treasure Island (FL): StatPearls Publishing; 2019 Jan.

2 Committee on Practice Bulletins-Obstetrics. ACOG Practice Bulletin No. 190: Gestational Diabetes Mellitus. Obstet Gynecol. 2018 Feb; 131(2):e49-64.

3 Cho NH, Shaw JE, Karuranga S, Huang Y, da Rocha Fernandes JD, Ohlrogge AW, et al. IDF Diabetes Atlas: global estimates of diabetes prevalence for 2017 and projections for 2045. Diabetes Res Clin Pract. 2018 Apr;138:27181.

4 Alfadhli EM. Gestational diabetes mellitus. Saudi Med J. 2015 Apr;36(4):399-406.

5 Mack LR, Tomich PG. Gestational Diabetes: Diagnosis, Classification, and Clinical Care. Obstet Gynecol Clin North Am. 2017 Jun; 44(2):207-17.

6 Aghajafari F, Nagulesapillai T, Ronksley PE, Tough SC, O’Beirne M, Rabi DM. Association between maternal serum 25-hydroxyvitamin $\mathrm{D}$ level and pregnancy and neonatal outcomes: systematic review and meta-analysis of observational studies. BMJ. 2013 Mar; 346:f1169.

$7 \mathrm{Lu} \mathrm{M}, \mathrm{Xu} \mathrm{Y,} \mathrm{Lv} \mathrm{L,} \mathrm{Zhang} \mathrm{M.} \mathrm{Association} \mathrm{be-}$ tween vitamin $\mathrm{D}$ status and the risk of gestational diabetes mellitus: a meta-analysis. Arch Gynecol Obstet. 2016 May;293(5):959-66.

8 Poel YH, Hummel P, Lips P, Stam F, van der Ploeg T, Simsek S. Vitamin D and gestational diabetes: a systematic review and meta-analysis. Eur J Intern Med. 2012 Jul;23(5):465-9.

9 Wei SQ, Qi HP, Luo ZC, Fraser WD. Maternal vitamin $\mathrm{D}$ status and adverse pregnancy outcomes: a systematic review and meta-analysis. J Matern Fetal Neonatal Med. 2013 Jun; 26(9):889-99.

10 Zhang MX, Pan GT, Guo JF, Li BY, Qin LQ, Zhang ZL. Vitamin D Deficiency Increases the Risk of Gestational Diabetes Mellitus: A Meta-Analysis of Observational Studies. Nutrients. 2015 Oct; 7(10):8366-75.

11 Sablok A, Batra A, Thariani K, Batra A, Bharti R, Aggarwal AR, et al. Supplementation of vitamin $\mathrm{D}$ in pregnancy and its correlation with feto-maternal outcome. Clin Endocrinol (Oxf). 2015 Oct;83(4):536-41.
12 Rostami M, Tehrani FR, Simbar M, Bidhendi Yarandi R, Minooee S, Hollis BW, et al. Effectiveness of Prenatal Vitamin D Deficiency Screening and Treatment Program: A Stratified Randomized Field Trial. J Clin Endocrinol Metab. 2018 Aug;103(8):2936-48.

13 IOM (Institute of Medicine). Dietary Reference Intakes for Calcium and Vitamin D. Washington (DC): The National Academies Press; 2011.

14 Gossman W, Chauhan K, Huecker MR. Vitamin D. StatPearls [Internet]. Treasure Island (FL): StatPearls Publishing; 2019.

15 Knabl J, Vattai A, Ye Y, Jueckstock J, Hutter S, Kainer F, et al. Role of Placental VDR Expression and Function in Common Late Pregnancy Disorders. Int J Mol Sci. 2017 Nov; 18(11).

16 Cho GJ, Hong SC, Oh MJ, Kim HJ. Vitamin $\mathrm{D}$ deficiency in gestational diabetes mellitus and the role of the placenta. Am J Obstet Gynecol. 2013 Dec;209(6):560.e1-8.

17 Knabl J, Hüttenbrenner R, Hutter S, Günthner-Biller M, Riedel C, Hiden U, et al. Gestational diabetes mellitus upregulates vitamin $\mathrm{D}$ receptor in extravillous trophoblasts and fetoplacental endothelial cells. Reprod Sci. 2015 Mar;22(3):358-66.

18 Jamilian M, Karamali M, Taghizadeh M, Sharifi N, Jafari Z, Memarzadeh MR, et al. Vitamin D and Evening Primrose Oil Administration Improve Glycemia and Lipid Profiles in Women with Gestational Diabetes. Lipids. 2016 Mar;51(3):349-56.

19 Karamali M, Asemi Z, Ahmadi-Dastjerdi M, Esmaillzadeh A. Calcium plus vitamin D supplementation affects pregnancy outcomes in gestational diabetes: randomized, doubleblind, placebo-controlled trial. Public Health Nutr. 2016 Jan;19(1):156-63.

20 Karamali M, Bahramimoghadam S, Sharifzadeh F, Asemi Z. Magnesium-zinc-calciumvitamin $\mathrm{D}$ co-supplementation improves glycemic control and markers of cardiometabolic risk in gestational diabetes: a randomized, double-blind, placebo-controlled trial. Appl Physiol Nutr Metab. 2018 Jun;43(6):565-70.
21 Jamilian M, Mirhosseini N, Eslahi M, Bahmani F, Shokrpour M, Chamani M, et al. The effects of magnesium-zinc-calcium-vitamin $\mathrm{D}$ co-supplementation on biomarkers of inflammation, oxidative stress and pregnancy outcomes in gestational diabetes. BMC Pregnancy Childbirth. 2019 Mar;19(1):107.

22 Kim SY, England L, Wilson HG, Bish C, Satten GA, Dietz P. Percentage of gestational diabetes mellitus attributable to overweight and obesity. Am J Public Health. 2010 Jun; 100(6): 1047-52.

23 Hedderson MM, Gunderson EP, Ferrara A. Gestational weight gain and risk of gestational diabetes mellitus. Obstet Gynecol. 2010 Mar;115(3):597-604.

24 Macrì F, Pitocco D, di Pasquo E, Salvi S, Rizzi A, Di Leo M, et al. Gestational weight gain as an independent risk factor for adverse pregnancy outcomes in women with gestational diabetes. Eur Rev Med Pharmacol Sci. 2018 Jul;22(14):4403-10.

25 Gante I, Amaral N, Dores J, Almeida MC. Impact of gestational weight gain on obstetric and neonatal outcomes in obese diabetic women. BMC Pregnancy Childbirth. 2015 Oct;15(1):249.

26 Institute of Medicine (US) and National Research Council (US) Committee to Reexamine IOM Pregnancy Weight Guidelines; Rasmussen KM, Yaktine AL, editors. Weight Gain During Pregnancy: Reexamining the Guidelines. Washington (DC): National Academies Press; 2009.

27 Kennel KA, Drake MT, Hurley DL. Vitamin D deficiency in adults: when to test and how to treat. Mayo Clin Proc. 2010 Aug;85(8): $752-7$.

28 Moher D, Shamseer L, Clarke M, Ghersi D, Liberati A, Petticrew M, et al.; PRISMA-P Group. Preferred reporting items for systematic review and meta-analysis protocols (PRISMA-P) 2015 statement. Syst Rev. 2015 Jan;4(1): 1 .

29 Ouzzani M, Hammady H, Fedorowicz Z, Elmagarmid A. Rayyan-a web and mobile app for systematic reviews. Syst Rev. 2016 Dec; 5(1):210. 
30 Liberati A, Altman DG, Tetzlaff J, Mulrow C, Gøtzsche PC, Ioannidis JP, et al. The PRISMA statement for reporting systematic reviews and meta-analyses of studies that evaluate health care interventions: explanation and elaboration. J Clin Epidemiol. 2009 Oct; 62(10):e1-34.

31 Higgins JPT, Green S, editors. Cochrane Handbook for Systematic Reviews of Interventions [Internet]. Version 5.1.0 [updated March 2011]. Cochrane Collaboration; 2011. Available from: www.cochrane-handbook. org
32 Higgins JP, Thompson SG, Deeks JJ, Altman DG. Measuring inconsistency in meta-analyses. BMJ. 2003 Sep;327(7414):557-60.

33 Kahveci B, Melekoglu R, Evruke IC, Cetin C. The effect of advanced maternal age on perinatal outcomes in nulliparous singleton pregnancies. BMC Pregnancy Childbirth. 2018 Aug;18(1):343.
34 Shan D, Qiu PY, Wu YX, Chen Q, Li AL, Ramadoss S, et al. Pregnancy Outcomes in Women of Advanced Maternal Age: a Retrospective Cohort Study from China. Sci Rep. 2018 Aug;8(1):12239.

35 Atkins D, Best D, Briss PA, Eccles M, FalckYtter Y, Flottorp S, et al.; GRADE Working Group. Grading quality of evidence and strength of recommendations. BMJ. 2004 Jun; 328(7454):1490. 